\title{
SAMPLING MEASURES, MUCKENHOUPT HAMILTONIANS, AND TRIANGULAR FACTORIZATION
}

\author{
R. V. BESSONOV
}

Abstract. Let $\mu$ be an even measure on the real line $\mathbb{R}$ such that

$$
c_{1} \int_{\mathbb{R}}|f|^{2} d x \leqslant \int_{\mathbb{R}}|f|^{2} d \mu \leqslant c_{2} \int_{\mathbb{R}}|f|^{2} d x
$$

for all functions $f$ in the Paley-Wiener space $\mathrm{PW}_{a}$. We prove that $\mu$ is the spectral measure for the unique Hamiltonian $\mathcal{H}=\left(\begin{array}{cc}w & 0 \\ 0 & \frac{1}{w}\end{array}\right)$ on $[0, a]$ generated by a weight $w$ from the Muckenhoupt class $A_{2}[0, a]$. As a consequence of this result, we construct Krein's orthogonal entire functions with respect to $\mu$ and prove that every positive, bounded, invertible Wiener-Hopf operator on $[0, a]$ with real symbol admits triangular factorization.

\section{INTRODUCTION}

The classical Paley-Wiener space $\mathrm{PW}_{a}$ consists of entire functions of exponential type at most $a$ square summable on the real line, $\mathbb{R}$. A measure $\mu$ on $\mathbb{R}$ is called a sampling measure for the space $\mathrm{PW}_{a}$ if there exist positive constants $c_{1}, c_{2}$ such that

$$
c_{1} \int_{\mathbb{R}}|f|^{2} d x \leqslant \int_{\mathbb{R}}|f|^{2} d \mu \leqslant c_{2} \int_{\mathbb{R}}|f|^{2} d x, \quad f \in \mathrm{PW}_{a} .
$$

Let $\mathcal{H}$ be a regular Hamiltonian on $[0, a]$, that is, $\mathcal{H}$ is a mapping from $[0, a]$ to the set of $2 \times 2$ non-negative matrices with real entries such that trace $\mathcal{H}$ is a positive non-vanishing function in $L^{1}[0, a]$. Denote by $\Theta_{\mathcal{H}}=\Theta_{\mathcal{H}}(r, z)$ solution of the following Cauchy problem:

$$
J X^{\prime}(r)=z \mathcal{H}(r) X(r), \quad X:[0, a] \rightarrow \mathbb{C}^{2}, \quad X(0)=\left(\begin{array}{c}
1 \\
0
\end{array}\right), \quad z \in \mathbb{C} .
$$

It is known from a general theory of canonical Hamiltonian systems that for every measure $\mu$ satisfying (11) there exists a regular Hamiltonian $\mathcal{H}$ with $\int_{0}^{a} \sqrt{\operatorname{det} \mathcal{H}}=a$ such that $\mu$ is a spectral measure for problem (2). The latter means that the Weyl-Titchmarsh transform

$$
\mathcal{W}_{\mathcal{H}, a}: X \mapsto \frac{1}{\sqrt{\pi}} \int_{0}^{a}\left\langle\mathcal{H}(r) X(r), \Theta_{\mathcal{H}}(r, \bar{z})\right\rangle_{\mathbb{C}^{2}} d r, \quad z \in \mathbb{C}
$$

2010 Mathematics Subject Classification. Primary 34L05, Secondary 47 B35.

Key words and phrases. Canonical Hamiltonian system, Muckenhoupt weight, Inverse problem, Paley-Wiener space, Truncated Toeplitz operator, Triangular factorization.

The work is supported by RFBR grant mol_a_dk 16-31-60053 and by "Native towns", a social investment program of PJSC "Gazprom Neft". 
generated by solution $\Theta_{\mathcal{H}}$ of Cauchy problem (2) maps isometrically the space

$$
\begin{gathered}
L^{2}(\mathcal{H}, a)=\left\{X:[0, a] \rightarrow \mathbb{C}^{2}:\|X\|_{L^{2}(\mathcal{H}, a)}^{2}=\int_{0}^{a}\langle\mathcal{H}(r) X(r), X(r)\rangle_{\mathbb{C}^{2}} d r<\infty\right\} / \mathcal{K}(\mathcal{H}), \\
\mathcal{K}(\mathcal{H})=\{X: \mathcal{H}(t) X(t)=0 \text { for almost all } t \in[0, r]\}
\end{gathered}
$$

into the space $L^{2}(\mu)$. A general problem in the inverse spectral theory is to translate properties of a spectral measure $\mu$ into properties of the Hamiltonian $\mathcal{H}$ it generates.

Two essentially different cases of the above problem attracted much attention. If $\mu$ is a "small perturbation" of the Lebesgue measure on $\mathbb{R}$ (in the sense that the Fourier transform of $\mu$ restricted to the interval $[-a, a]$ differs from the point mass measure $\delta_{0}$ concentrated at 0 by a function in $\left.L^{1}[-a, a]\right)$, the I. M. GelfandB. M. Levitan approach [6], [12] gives a quite precise information on relation between $\mu$ and $\mathcal{H}$. On the other hand, if $\mu$ is arbitrary measure on $\mathbb{R}$ such that $\int_{\mathbb{R}} \frac{d \mu(t)}{1+t^{2}}<\infty$, the theory of M. G. Krein [8] (for even measures $\mu$ ) and L. de Branges [4] (for all $\mu$ ) implies the existence of a unique Hamiltonian $\mathcal{H} \in L_{\text {loc }}^{1}[0, \infty$ ) such that $\mu$ is the spectral measure for $\mathcal{H}$. However, it is not known how translate even simple properties of a Hamiltonian $\mathcal{H}$ (e.g., membership in $L^{p}$ class for some $p>1)$ to the properties of its spectral measure $\mu$ and vice versa. In this paper we consider a "median" situation (spectral measures with sampling property (1) for the Paley-Wiener space PW $\mathrm{PW}_{a}$ ) and use both Gelfand-Levitan and Krein-de Branges theories.

A measure $\mu$ on $\mathbb{R}$ is called even if $\mu(S)=\mu(-S)$ for every Borel set $S \subset \mathbb{R}$. A function $w>0$ belongs to the Muckenhoupt class $A_{2}[0, a]$ if the supremum of products $\left(\frac{1}{|I|} \int_{I} w\right) \cdot\left(\frac{1}{|I|} \int_{I} \frac{1}{w}\right)$ over all intervals $I \subset[0, a]$ is finite. Here is the main result of the paper.

Theorem 1. Let $\mu$ be an even sampling measure for $\mathrm{PW}_{a}$. Then $\mu$ is the spectral measure for problem (2) corresponding to the unique Hamiltonian $\mathcal{H}=\left(\begin{array}{cc}w & 0 \\ 0 & \frac{1}{w}\end{array}\right)$ generated by a weight $w \in A_{2}[0, a]$.

The Hamiltonian $\mathcal{H}$ in Theorem 1 could be recovered from the spectral measure $\mu$ by means of the following simple formula:

$$
w(r)=\pi \frac{\partial}{\partial r}\left\|T_{\mu, r}^{-1} \operatorname{sinc}_{r}\right\|_{L^{2}(\mu)}^{2}, \quad \operatorname{sinc}_{r}=\frac{\sin r x}{\pi x}, \quad r \in[0, a],
$$

where $T_{\mu, r}$ is the truncated Toeplitz operator on $\mathrm{PW}_{r}$ with symbol $\mu$ defined by

$$
\left(T_{\mu, r} f\right)(z)=\int_{\mathbb{R}} f(x) \frac{\sin r(x-z)}{\pi(x-z)} d \mu(x), \quad z \in \mathbb{C} .
$$

A nontrivial fact is that the continuous increasing function $r \mapsto\left\|T_{\mu, r}^{-1} \operatorname{sinc}_{r}\right\|_{L^{2}(\mu)}^{2}$ is absolutely continuous and its derivative $w / \pi$ does not vanish on a set of positive Lebesgue measure. In the proof of Theorem 1 we first obtain an estimate for the " $A_{2}$-norm" of $w$ in terms of $c_{1}, c_{2}$ assuming above properties of $w$; then use an approximation argument based on a description of positive truncated Toeplitz operators on $\mathrm{PW}_{r}$ and $L^{p}$-summabilty of weights $w \in A_{2}[0, a]$ for some $p>1$.

Section 5 in $[2$ contains an example of a diagonal Hamiltonian $\mathcal{H}$ on $[0,1]$ such that both $\mathcal{H}, \mathcal{H}^{-1}$ are uniformly bounded on $[0,1]$, but the spectral measures of the corresponding problem (2) fail to have sampling property. This shows that $A_{2}[0, a]$ 
class does not describe canonical Hamiltonian systems generated by sampling measures for $\mathrm{PW}_{a}$.

Theorem 1 yields two results of independent interest.

Given a measure $\mu$ satisfying (1) and a number $r \in[0,2 a]$, denote by $\left(\mathrm{PW}_{[0, r]}, \mu\right)$ the Paley-Wiener space of functions from $L^{2}(\mathbb{R})$ with Fourier spectrum in $[0, r]$ equipped with the inner product taken from $L^{2}(\mu)$.

Theorem 2. Let $\mu$ be an even sampling measure for the space $\mathrm{PW}_{a}$. Then there exists a family of entire functions $\left\{P_{t}\right\}_{t \in[0,2 a]}$ such that $\mathcal{F}_{\mu}: f \mapsto \frac{1}{\sqrt{2 \pi}} \int_{0}^{r} f(t) P_{t}(z) d t$ is the unitary operator from $L^{2}[0, r]$ to $\left(\mathrm{PW}_{[0, r]}, \mu\right)$ for every $r \in[0,2 a]$.

In the case where $\mu$ is a "small perturbation" of the Lebesgue measure (see discussion above), the functions $P_{r}$ in Corollary 2 coincide with orthogonal entire functions constructed by M. G. Krein in [10. S. A. Denisov provides an extensive treatment of the subject, collecting many old and new results in paper [5].

The second application of Theorem 1 concerns the classical factorization problem for positive invertible operators. Let $H$ be a separable Hilbert space and let $B(H)$ be the algebra of all bounded operators on $H$. Consider a complete chain $\mathcal{N}$ of subspaces in $H$ and denote by $\mathcal{A}_{\mathcal{N}}=\{A \in B(H): A E \subset E, E \in \mathcal{N}\}$ the nest algebra of upper-triangular operators with respect to $\mathcal{N}$. In sixties, I. C. Gohberg and M. G. Krein proved (see Theorem 6.2 in Chapter 4 of [7]) that every positive invertible operator $T$ on $H$ of the form $T=I-K$ with $K$ in Macaev ideal $S_{\omega}$ admits the triangular factorization $T=A^{*} A$, where $A=I-K_{A}$ is an invertible operator on $H$ such that $K_{A} \in S_{\omega} \cap \mathcal{A}_{\mathcal{N}}$. Famous theorem by D. R. Larson [11] says that every positive invertible operator $T$ admits triangular factorization $T=A^{*} A$ with $A, A^{-1} \in \mathcal{A}_{\mathcal{N}}$ if and only if the chain $\mathcal{N}$ is countable. Moreover, given $0<\varepsilon<1$, the non-factorable operator $T$ can be chosen so that $K=I-T$ is a compact operator with $\|K\|<\varepsilon$.

We consider the problem of triangular factorization for Wiener-Hopf convolution operators. Let $\psi \in \mathcal{S}^{\prime}$ be a tempered distribution on $\mathbb{R}$ and let $0<a \leqslant \infty$. The Wiener-Hopf operator $W_{\psi}$ on $L^{2}[0, a)$ with symbol $\psi$ is densely defined by

$$
\left(W_{\psi} f\right)(y)=\left\langle\psi, s_{y} f\right\rangle_{\mathcal{S}^{\prime}}, \quad y \in[0, a), \quad s_{y} f: x \mapsto f(x-y),
$$

on smooth functions $f$ with compact support in $(0, a)$. In the case where $\psi \in L^{1}(\mathbb{R})$ we have more familiar definition, $W_{\psi}: f \mapsto \int_{0}^{a} \psi(x-y) f(x) d x$. As following result shows, Wiener-Hopf operators with real symbols are always factorable.

Theorem 3. Let $0<a \leqslant \infty$. Every positive, bounded, and invertible Wiener-Hopf operator $W_{\psi}$ on $L^{2}[0, a)$ with real symbol $\psi \in \mathcal{S}^{\prime}$ admits triangular factorization: $W_{\psi}=A^{*} A$, where $A$ is a bounded invertible operator such that $A L^{2}[0, r]=L^{2}[0, r]$ for every $r \in[0, a)$.

Wiener-Hopf operators $W_{\psi}$ in Theorem 3 admit triangular factorizations in the reverse order $W_{\psi}=A A^{*}$ as well. Relation of absolute continuity of aforementioned function $r \mapsto\left\|T_{\mu, r}^{-1} \operatorname{sinc}_{r}\right\|_{L^{2}(\mu)}^{2}$ to triangular factorization problems has been previously found in different terms by L. A. Sakhnovich, see Theorem 4.2 in [16]. On the other hand, Theorem 3 contradicts Theorem 4.1 from another work [17] by the same author. See discussion in Section 5 . 
Acknowledgement. The author is grateful to many colleagues who took a part in discussions related to the subject of the paper, especially to Roman Romanov, Mikhail Sodin, Pavel Zatitsky, and Dmitriy Zaporozhets.

\section{Integration over Simplex and the Muckenhoupt Class $A_{2}$}

Let $w$ be a positive function on an interval $[0, a]$. We associate to $w$ the quantity

$$
\|w\|_{A_{2}[0, a]}=\sup _{I \subset[0, a]}\left(\frac{1}{|I|} \int_{I} w(x) d x\right) \cdot\left(\frac{1}{|I|} \int_{I} \frac{1}{w(x)} d x\right),
$$

where $I$ runs over all subintervals of $[0, a]$. Note that $\|\cdot\|_{A_{2}[0, a]}$ is not a norm in the standard sense, but we will use this convenient notation. The Muckenhoupt class $A_{2}[0, a]$ consists of functions $w>0$ such that $\|w\|_{A_{2}[0, a]}<\infty$. In this section we present a special integral condition for a weight $w$ to belong to the $A_{2}[0, a]$ class.

Let $\varphi$ be a real-valued function on the interval $[0, a]$. For a real $0<t<a$ and an integer $n \geqslant 1$ define the mapping

$$
G_{\varphi, n}: x \mapsto \sum_{k=1}^{n}(-1)^{n+k} \varphi\left(x_{k}\right), \quad x \in K_{t, n},
$$

on simplex $K_{t, n}=\left\{x \in \mathbb{R}^{n}: x=\left(x_{1}, \ldots, x_{n}\right), t \geqslant x_{1} \geqslant \ldots \geqslant x_{n} \geqslant 0\right\}$. Let $m_{n}$ denote the usual Lebesgue measure on $\mathbb{R}^{n}$.

Next proposition will be used in the proof of Theorem 1

Proposition 2.1. Let $\varphi$ be a function on $[0, a]$ such that $e^{|\varphi|} \in L^{1}[0, a]$. Assume that for every $r \in[0, a]$ and every integer $n \geqslant 1$ we have

$$
\begin{gathered}
\frac{1}{a_{n}(r)} \int_{0}^{r} e^{(-1)^{n} \varphi(t)}\left(\int_{K_{t, n}} e^{G_{\varphi, n}(x)} d m_{n}(x)\right)^{2} d t \leqslant b_{2}, \\
b_{1} \leqslant \frac{1}{r} \int_{0}^{r} e^{\varphi(t)} d t \leqslant b_{2}
\end{gathered}
$$

where $b_{1}, b_{2}$ are positive constants, and $a_{n}(r)=r^{2 n+1}(2 n+1)^{-1}(n !)^{-2}$. Then the function $w=e^{\varphi}$ belongs to $A_{2}[0, a]$ and $\|w\|_{A_{2}[0, a]} \leqslant 2^{28}\left(b_{2}+b_{1}^{-2} b_{2}\right)^{14}$.

We first prove several preliminary estimates.

Lemma 2.1. Let $\varphi$ be a function as in Proposition 2.1. Then for every $r \in[0, a]$ and $b=2\left(b_{2}+b_{1}^{-2} b_{2}\right)$ we have

$$
\frac{1}{r} \int_{0}^{r}|\varphi(t)| d t \leqslant \log b, \quad \frac{1}{r} \int_{0}^{r} e^{|\varphi(t)|} d t \leqslant b .
$$

Consequently, for every decreasing differentiable function $k \geqslant 0$ on $[0, r]$ satisfying $\int_{0}^{r} k(t) d t=1$ and $k(r)=0$ we have $\int_{0}^{r}|\varphi(t)| k(t) d t \leqslant \log b$.

Proof. Clearly, the first estimate in (8) follows from the second one and the Jensen's inequality for convex function $e^{x}$. Taking $n=1$ in (6), we obtain

$$
\frac{3}{r^{3}} \int_{0}^{r} e^{-\varphi(t)}\left(\int_{0}^{t} e^{\varphi\left(t_{1}\right)} d t_{1}\right)^{2} d t \leqslant b_{2} .
$$


From (77) we know that $\frac{1}{t} \int_{0}^{t} e^{\varphi\left(t_{1}\right)} d t_{1} \geqslant b_{1}$ for all $t \in[0, r]$. It follows that

$$
b_{1}^{-2} b_{2} \geqslant \frac{3}{r^{3}} \int_{0}^{r} e^{-\varphi(t)} t^{2} d t \geqslant \frac{1}{r} \int_{r / 2}^{r} e^{-\varphi(t)} d t
$$

Using the other side estimate $\frac{1}{r} \int_{0}^{r} e^{\varphi(t)} d t \leqslant b_{2}$ and inequality $e^{|x|} \leqslant e^{x}+e^{-x}$, we see that

$$
\frac{2}{r} \int_{r / 2}^{r} e^{|\varphi(t)|} d t \leqslant b_{2}+b_{1}^{-2} b_{2}
$$

for all $r \in[0, a]$. Then (8) follows from

$$
\frac{1}{r} \int_{0}^{r} e^{|\varphi(t)|} d t=\frac{1}{r}\left(\sum_{k=0}^{\infty}\left|I_{r, k}\right| \cdot \frac{1}{\left|I_{r, k}\right|} \int_{I_{r, k}} e^{|\varphi(t)|} d t\right) \leqslant b
$$

where $I_{r, k}=\left[2^{-k-1} r, 2^{-k} r\right]$. Now if $k$ is a function on $[0, r] \subset[0, a]$ as in the statement, we have

$$
\begin{aligned}
\int_{0}^{r}|\varphi(t)| k(t) d t & =-\int_{0}^{r}|\varphi(t)| \int_{0}^{r} \chi_{[t, r]}(s) k^{\prime}(s) d s d t \\
& =-\int_{0}^{r} k^{\prime}(s) \int_{0}^{r} \chi_{[0, s]}(t)|\varphi(t)| d t d s \\
& \leqslant-\log b \int_{0}^{r} k^{\prime}(s) s d s=\log b
\end{aligned}
$$

This completes the proof.

For $n \geqslant 1$ introduce the intervals $I_{t, n}=\left[\delta_{n} t, t\right]$, where $\delta_{n}=1-\frac{1}{n+1}$ if $n$ is odd, and $\delta_{n}=1-\frac{1}{n}$ if $n$ is even. In particular, $I_{t, n}=I_{t, n+1}$ for every odd $n$. Set

$$
[\varphi]_{t, n}=2(-1)^{n+1} \int_{K_{t, n}} G_{\varphi, n}(x) d m_{t, n}(x)
$$

where $m_{t, n}=\frac{n !}{t^{n}} \cdot m_{n}$ is the scalar multiple of the Lebesgue measure $m_{n}$ on $\mathbb{R}^{n}$ normalized so that $m_{t, n}\left(K_{t, n}\right)=1$.

Lemma 2.2. For $r \in[0, a]$ and odd $n \geqslant 1$ we have $\left|[\varphi]_{\delta_{n} r, n}-[\varphi]_{\delta_{n+1} r, n+1}\right|<6 \log b$, where $b$ is the constant from Lemma 2.1.

Proof. Arguing by induction, it is easy check that for all $n \geqslant 1$ and $\tau \in[0, a]$ we have

$$
[\varphi]_{\tau, n}=\int_{0}^{\tau} \varphi(s) k_{\tau, n}(s) d s, \quad k_{\tau, n}(s)=\frac{2 n}{\tau^{n}}(2 s-\tau)^{n-1} .
$$

For odd (correspondingly, even) integers $n$ the kernels $k_{\tau, n}$ are even (correspondingly, odd) functions with respect to the point $\tau / 2$. As $n$ tends to infinity, the kernels $k_{\tau, n}$ tend to zero uniformly on every closed interval in $(0, \tau)$. We also have

$$
\int_{0}^{\tau}\left|k_{\tau, n}(s)\right| d s=2, \quad \sup _{s \in[\tau / 2, \tau]}\left|k_{\tau, n}(s)-k_{\tau, n+1}(s)\right| \leqslant \frac{2}{\tau} .
$$


Now take an odd integer $n \geqslant 1$ and note that $\delta_{n}=\delta_{n+1}=\frac{1}{n+1}$. Setting $\tau=\delta_{n} r$, we obtain

$$
\begin{aligned}
\left|[\varphi]_{\tau, n}-[\varphi]_{\tau, n+1}\right| \leqslant & \int_{0}^{\tau / 2}\left|\varphi(s) k_{\tau, n}(s)\right| d s \\
& +\int_{0}^{\tau / 2}\left|\varphi(s) k_{\tau, n+1}(s)\right| d s \\
& +\int_{\tau / 2}^{\tau}|\varphi(s)| \cdot\left|k_{\tau, n}(s)-k_{\tau, n+1}(s)\right| d s .
\end{aligned}
$$

By Lemma 2.1 for functions $\varphi, k=\frac{1}{2}\left|k_{\tau, n}\right|$, and $k=\frac{1}{2}\left|k_{\tau, n+1}\right|$ on $\left[0, \frac{\tau}{2}\right]$, the sum of first two integrals is bounded from above by $4 \log b$. To show that the last integral does not exceed $2 \log b$, use (8) and the second estimate in (9).

Proof of Proposition 2.1. Take an odd integer $n \geqslant 1$. Since the integrand in (6) is positive, we have

$$
\begin{aligned}
b_{2} & \geqslant \frac{1}{a_{n}(r)} \int_{\delta_{n} r}^{r} e^{-\varphi(t)}\left(\int_{K_{t, n}} e^{G_{\varphi, n}(x)} d m_{n}(x)\right)^{2} d t \\
& \geqslant \frac{1}{a_{n}(r)}\left(\int_{\delta_{n} r}^{r} e^{-\varphi(t)} d t\right) \cdot\left(\int_{K_{\delta_{n} r, n}} e^{G_{\varphi, n}(x)} d m_{n}(x)\right)^{2} .
\end{aligned}
$$

By Jensen's inequality,

$$
\int_{K_{\delta_{n} r, n}} e^{G_{\varphi, n}(x)} d m_{n}(x) \geqslant \frac{\left(\delta_{n} r\right)^{n}}{n !} \exp \left(\frac{[\varphi]_{\delta_{n} r, n}}{2}\right) .
$$

For all $n \geqslant 1$ we have

$$
\frac{1}{a_{n}(r)} \cdot\left(\frac{\left(\delta_{n} r\right)^{n}}{n !}\right)^{2}=\frac{(2 n+1)(n !)^{2}}{r^{2 n+1}} \cdot \frac{r^{2 n}}{(n !)^{2}} \delta_{n}^{2 n} \geqslant \frac{n+1}{32 r}=\frac{1}{32\left|I_{n, r}\right|} .
$$

We now see that

$$
\frac{1}{\left|I_{r, n}\right|} \int_{I_{r, n}} \exp \left(-\varphi(t)+[\varphi]_{\delta_{n} r, n}\right) d t \leqslant 32 b_{2} .
$$

Analogously, for the even integer $n+1$ we have

$$
\frac{1}{\left|I_{r, n+1}\right|} \int_{I_{r, n+1}} \exp \left(\varphi(t)-[\varphi]_{\delta_{n+1} r, n}\right) d t \leqslant 32 b_{2}
$$

Recall that $I_{t, n+1}=I_{t, n}$. Applying Lemma 2.2, we obtain

$$
\frac{1}{\left|I_{r, n}\right|} \int_{I_{r, n}} \exp \left(\varphi(t)-[\varphi]_{\delta_{n} r, n}\right) d t \leqslant 32 b_{2} e^{6 \log b} \leqslant 32 b^{7}
$$

where $b$ is the constant from Lemma 2.1 Using inequality $e^{|x|} \leqslant e^{x}+e^{-x}$, we get from (10) and (11) the estimate

$$
\frac{1}{|I|} \int_{I} e^{\left|\varphi(t)-c_{I}\right|} d t \leqslant 64 b^{7}
$$

for all intervals $I$ of the form $I=\left[\left(1-\frac{1}{n+1}\right) r, r\right]$, where $r \in[0, a]$, and integer $n \geqslant 1$ is odd. Here $c_{I}$ is a constant depending on $I$ (in fact, $c_{I}=[\varphi]_{\delta_{n} r, n}$ works, but from now on the particular choice of $c_{I}$ plays no role). Formula (8) gives (12) with $c_{I}=0$ for intervals of the form $I=[0, t]$. 
Next, observe that each interval $J \subset[0, a]$ is contained in an interval $I$ satisfying (12) and such that $|I| \leqslant 2|J|$. Indeed, let $t$ be the right point of $J$. If $|J| \geqslant|t| / 2$, take $I=[0, t]$. In the case $|J|<|t| / 2$ find an odd number $n \geqslant 1$ such that $I_{t, n+2} \subset J \subset I_{t, n}$ and take $I=I_{t, n}$. Fix this interval $I$ and the corresponding constant $c_{I}$ form (12). We have

$$
\begin{aligned}
\left(\frac{1}{|J|} \int_{J} e^{\varphi} d t\right) \cdot\left(\frac{1}{|J|} \int_{J} e^{-\varphi} d t\right) & \leqslant\left(\frac{2}{|I|} \int_{I} e^{\varphi} d t\right) \cdot\left(\frac{2}{|I|} \int_{I} e^{-\varphi} d t\right) \\
& \leqslant\left(\frac{2}{|I|} \int_{I} e^{\varphi-c_{I}} d t\right) \cdot\left(\frac{2}{|I|} \int_{I} e^{-\varphi+c_{I}} d t\right) \leqslant(2 b)^{14}
\end{aligned}
$$

Since interval $J$ is arbitrary, this shows that function $w=e^{\varphi}$ belongs to the Muckenhoupt class $A_{2}[0, a]$ and $\|w\|_{A_{2}[0, a]} \leqslant(2 b)^{14}=2^{28}\left(b_{2}+b_{1}^{-2} b_{2}\right)^{14}$.

\section{Proof of Theorem 1}

As it was mentioned in the Introduction, we will use an approximation argument in the proof of Theorem 11 To have a stable approximation, we need a result describing positive truncated Toeplitz operators on $\mathrm{PW}_{a}$.

3.1. Preliminaries on truncated Toeplitz operators. Let $\mu \geqslant 0$ be a measure on the real line $\mathbb{R}$ such that $\|f\|_{L^{2}(\mu)}^{2} \leqslant c\|f\|_{L^{2}(\mathbb{R})}^{2}$ for all functions $f \in \mathrm{PW}_{[0, a]}$. Define the truncated Toeplitz operator $A_{\mu, a}$ on $\mathrm{PW}_{[0, a]}$ by the sesquilinear form

$$
\left(A_{\mu, a} f, g\right)_{L^{2}(\mathbb{R})}=\int_{\mathbb{R}} f \bar{g} d \mu, \quad f, g \in \mathrm{PW}_{[0, a]} .
$$

In the case where $\mu=u d m$ is absolutely continuous with respect to the Lebesgue measure $m$ on $\mathbb{R}$ and has density $u$, the operator $A_{\mu, a}$ coincides with the projection of the standard Toeplitz operator $T_{u}$ on the Hardy space $H^{2}$ to the subspace $\mathrm{PW}_{[0, a]}$. This explains the name "truncated Toeplitz" for the operator $A_{\mu, a}$.

It is well-known (see, e.g., Section 6.1 in [14]) that the operator

$$
V: h \mapsto \frac{1}{\sqrt{\pi}} \frac{1}{z+i} h\left(\frac{z-i}{z+i}\right), \quad z \in \mathbb{C}_{+},
$$

maps unitarily the Hardy space $H^{2}(\mathbb{D})$ in the open unit disk $\mathbb{D}=\{\xi \in \mathbb{C}:|\xi|<1\}$ onto the Hardy space $H^{2}$ in the upper half-plane $\mathbb{C}_{+}=\{z \in \mathbb{C}: \operatorname{Im} z>0\}$. Moreover, for every $a>0$ we have $V K_{\theta_{a}}=\mathrm{PW}_{[0, a]}$, where $\theta_{a}=\exp \left(a \frac{z+1}{z-1}\right)$ is the inner function in $\mathbb{D}$ and $K_{\theta_{a}}$ is the orthogonal complement in $H^{2}(\mathbb{D})$ to the subspace $\theta_{a} H^{2}(\mathbb{D})$. As we will see in a moment, the truncated Toeplitz operators defined by (13) are unitarily equivalent to truncated Toeplitz operators on the shiftcoinvariant subspace $K_{\theta_{a}}$ of $H^{2}(\mathbb{D})$. See D. Sarason's paper [18] for basic properties of truncated Toeplitz operators on general coinvariant subspaces of $H^{2}(\mathbb{D})$.

We also will deal with the operators $T_{\mu, a}$ on the space $\mathrm{PW}_{a}$ defined by the same sesquilinear form

$$
\left(T_{\mu, a} f, g\right)=\int_{\mathbb{R}} f \bar{g} d \mu, \quad f, g \in \mathrm{PW}_{a} .
$$

It is easy to see that this definition agrees with formula (4). By construction, we have $T_{\mu, a}=V_{a}^{-1} A_{\mu, 2 a} V_{a}$, where $V_{a}: \mathrm{PW}_{a} \rightarrow \mathrm{PW}_{[0,2 a]}$ is the unitary operator taking a function $f$ into $e^{i a z} f$. 
Lemma 3.1. Let $T$ be a positive bounded operator on $\mathrm{PW}_{[0, a]}$ satisfying relation

$$
(T f, f)_{L^{2}(\mathbb{R})}=\left(T \frac{z-i}{z+i} f, \frac{z-i}{z+i} f\right)_{L^{2}(\mathbb{R})}
$$

for all functions $f \in \mathrm{PW}_{[0, a]}$ such that $f(-i)=0$. Then there exists a positive measure $\mu$ on $\mathbb{R}$ such that $T=A_{\mu, a}$. Similarly if $T$ is a positive bounded operator on $\mathrm{PW}_{a}$ satisfying (15) for all $f \in \mathrm{PW}_{a}$ such that $f(-i)=0$, then $T=T_{\mu, a}$ for a positive measure $\mu$ on $\mathbb{R}$.

Proof. Let $\theta_{a}, K_{\theta_{a}}$, and $V: K_{\theta_{a}} \rightarrow \mathrm{PW}_{[0, a]}$ be defined as above. Consider the operator $\tilde{T}=V^{-1} T V$ on $K_{\theta_{a}}$ unitarily equivalent to the operator $T$ on $\mathrm{PW}_{[0, a]}$. Recall that the inner product in $K_{\theta_{a}}$ is inherited from the space $L^{2}(\mathbb{T})$ on the unit circle $\mathbb{T}=\{\xi \in \mathbb{C}:|\xi|=1\}$. Assumption (15) means that

$$
(\tilde{T} h, h)_{L^{2}(\mathbb{T})}=(\tilde{T} \xi h, \xi h)_{L^{2}(\mathbb{T})}
$$

for every function $h \in K_{\theta_{a}}$ such that $\xi h \in K_{\theta_{a}}$. Indeed, $(V \xi h)(z)=\frac{z-i}{z+i}(V h)(z)$ and hence $V(\xi h) \in \mathrm{PW}_{[0, a]}$ if and only if $(V h)(-i)=0$. Theorem 8.1 in [18] says that a bounded operator $\tilde{T}$ on $K_{\theta_{a}}$ (or on any other coinvariant subspace $K_{\theta}^{2}$ of the Hardy space $H^{2}(\mathbb{D})$ ) satisfying (16) is a truncated Toeplitz operator on $K_{\theta_{a}}$. By Theorem 2.1 in [1], for every positive bounded truncated Toeplitz operator $\tilde{T}$ on $K_{\theta_{a}}$ there exists a finite positive measure $\tilde{\mu}$ on $\mathbb{T}$ such that $\tilde{\mu}(\{1\})=0$ and

$$
(\tilde{T} h, h)_{L^{2}(\mathbb{T})}=\int_{\mathbb{T}}|h|^{2} d \tilde{\mu}
$$

for all continuous functions $h$ in $K_{\theta_{a}}$. Changing variables in the last integral, we find a positive measure $\mu$ on $\mathbb{R}$ such that

$$
\int_{\mathbb{T}}|h|^{2} d \tilde{\mu}=\int_{\mathbb{R}}|f|^{2} d \mu, \quad f=V h .
$$

It follows that $(T f, f)=(\tilde{T} h, h)_{L^{2}(\mathbb{T})}=\left(A_{\mu, a} f, f\right)_{L^{2}(\mathbb{R})}$ for a dense set of functions $f$ in $\mathrm{PW}_{[0, a]}$. Since $T$ is continuous, we have $T=A_{\mu, a}$. The second part of the Lemma is a direct consequence of relation $T_{\mu, a}=V_{a}^{-1} A_{\mu, 2 a} V_{a}$.

3.2. Preliminaries on canonical Hamiltonian systems. Let $\mathcal{H}$ be a Hamiltonian on $[0, a]$ with trace $\mathcal{H} \in L^{1}[0, a]$. Assume that there is no interval $\left(r_{1}, r_{2}\right) \subset[0, a]$ such that $\mathcal{H}(t)$ is a constant matrix of rank one for all points $t \in\left(r_{1}, r_{2}\right)$. For $r \in[0, a]$ we will denote by $\mathcal{B}(\mathcal{H}, r)$ the de Branges space generated by $\mathcal{H}$ on $[0, r]$, that is,

$$
\mathcal{B}(\mathcal{H}, r)=\mathcal{W}_{\mathcal{H}, r} L^{2}(\mathcal{H}, r)=\left\{\text { entire } f: f=\mathcal{W}_{\mathcal{H}, r} X, \quad X \in L^{2}(\mathcal{H}, r)\right\},
$$

where the Weyl-Titchmarsh transform $\mathcal{W}_{\mathcal{H}, r}$ is defined in (3) for $a=r$. The space $\mathcal{B}(\mathcal{H}, r)$ is actually the Hilbert space with respect to the inner product $(f, g)_{\mathcal{B}(\mathcal{H}, r)}=(f, g)_{L^{2}(\mu)}$, where $\mu$ is any spectral measure for problem (2). We refer the reader to paper [2] for the summary of results on direct and inverse spectral theory of canonical Hamiltonian systems and de Brange spaces of entire functions. The readers interested in proofs or in a more detailed account may find necessary information in Chapter 2 of classical book 4 by L. de Brange or its recent exposition [15] by R. Romanov. 
Lemma 3.2. Let $\mu$ be an even measure on $\mathbb{R}$ of the form $\mu=c m+\nu$, where $c>0$ and $\nu$ is a finite positive measure on $\mathbb{R}$ with compact support. Then there exists an infinitely smooth diagonal Hamiltonian $\mathcal{H}$ on $[0,+\infty)$ such that $\operatorname{det} \mathcal{H}(r)=1$ for all $r \geqslant 0$, and $\mu$ is the spectral measure for $\mathcal{H}$.

Proof. The result is a kind of folklore. Since the Fourier transform of $\frac{1}{c} \nu$ is a smooth (in fact, analytic) function, one can use the classical Gelfand-Levitan approach to find a smooth diagonal potential $Q$ on $[0, a]$ such that $m+\frac{1}{c} \nu$ is the spectral measure for the Dirac system $J Y^{\prime}+Q Y=z Y$ corresponding to the boundary condition $Y(0)=\left(\begin{array}{l}1 \\ 0\end{array}\right)$. Then rewrite system $J Y^{\prime}+Q Y=z Y$ as a canonical Hamiltonian system $J X^{\prime}=z \tilde{\mathcal{H}} X$ setting $X=M^{-1} Y, \tilde{\mathcal{H}}=M^{*} M$, where $M$ is the matrix solution of equation $J M^{\prime}=-Q M, M(0)=\left(\begin{array}{ll}1 & 0 \\ 0 & 1\end{array}\right)$. Observe that $\operatorname{det} \tilde{\mathcal{H}}=1$ almost everywhere on $[0, a]$ and $m+\frac{1}{c} \nu$ is the spectral measure for system $J X^{\prime}=z \tilde{\mathcal{H}} X, X(0)=\left(\begin{array}{l}1 \\ 0\end{array}\right)$. To obtain the Hamiltonian on $[0, a]$ corresponding to the spectral measure $\mu$, put $\mathcal{H}=\left(\begin{array}{cc}c & 0 \\ 0 & \frac{1}{c}\end{array}\right) \tilde{\mathcal{H}}$. Another (in a sense, equivalent) way of proving Lemma 3.2 is the application of Theorem 5.1 from [20].

Define type $\mathcal{B}(\mathcal{H}, r)=\sup \{\operatorname{type}(f), f \in \mathcal{B}(\mathcal{H}, r)\}$ to be the maximal exponential type of entire functions in de Branges space $\mathcal{B}(\mathcal{H}, r)$. The following remarkable formula of Krein [9] and de Brange (Theorem X in [3])

$$
\text { type } \mathcal{B}(\mathcal{H}, r)=\int_{0}^{r} \sqrt{\operatorname{det} \mathcal{H}(t)} d t
$$

represents the maximal exponential type of functions in $\mathcal{B}(\mathcal{H}, r)$ in terms of the Hamiltonian $\mathcal{H}$. Section 6 in 15 contains an elegant self-contained proof of this result.

Lemma 3.3. Let $\mathcal{H}$ be a Hamiltonian on an interval $[0, a]$ such that its spectral measure $\mu$ satisfies (11). Assume that $\operatorname{det} \mathcal{H}(r)=1$ for almost all $r \in[0, a]$. Then for all $r \in[0, a]$ we have $\mathcal{B}(\mathcal{H}, r)=\left(\mathrm{PW}_{r}, \mu\right)$.

Proof. Let $r \in[0, a)$ and let $\varepsilon>0$ be such that $r \in[\varepsilon, a-\varepsilon)$. Then the Hilbert space $\left(\mathrm{PW}_{r+\varepsilon}, \mu\right)$ of entire functions satisfies an axiomatic description of de Branges spaces (Theorem 23 in [4) and the embedding $\left(\mathrm{PW}_{r+\varepsilon}, \mu\right) \subset L^{2}(\mu)$ is isometric. Since $\mu$ is a spectral measure for $\mathcal{H}$, the embedding $\mathcal{B}(\mathcal{H}, r) \subset L^{2}(\mu)$ is isometric as well. Applying de Branges chain theorem (Theorem 35 in [4]), we see that ether $\left(\mathrm{PW}_{r+\varepsilon}, \mu\right) \subset \mathcal{B}(\mathcal{H}, r)$ or $\mathcal{B}(\mathcal{H}, r) \subset\left(\mathrm{PW}_{r+\varepsilon}, \mu\right)$. Since det $\mathcal{H}=1$ almost everywhere on $[0, a]$, formula (17) implies the second alternative. Analogously, one can show that $\left(\mathrm{PW}_{r-\varepsilon}, \mu\right) \subset \mathcal{B}(\mathcal{H}, r)$. Since this holds for every small number $\varepsilon$ and $\mu$ is sampling, we have $\mathcal{B}(\mathcal{H}, r)=\left(\mathrm{PW}_{r}, \mu\right)$. Finally, for $r=a$ we have

$$
\mathcal{B}(\mathcal{H}, a)=\overline{\bigcup_{0<r<a} \mathcal{B}(\mathcal{H}, r)}=\left(\mathrm{PW}_{a}, \mu\right),
$$

where the completion is taken with respect to the norm inherited from $L^{2}(\mu)$.

Let $\Theta_{\mathcal{H}}$ be the absolutely continuous solution of Cauchy problem (2) on $[0, a]$, and denote $\Theta_{\mathcal{H}}^{+}=\left\langle\Theta_{\mathcal{H}},\left(\begin{array}{l}1 \\ 0\end{array}\right)\right\rangle, \Theta_{\mathcal{H}}^{-}=\left\langle\Theta_{\mathcal{H}},\left(\begin{array}{l}0 \\ 1\end{array}\right)\right\rangle$. The reproducing kernel $k_{\mathcal{B}(\mathcal{H}, r) ; \lambda}$ at a point $\lambda \in \mathbb{C}$ of the Hilbert space of entire functions $\mathcal{B}(\mathcal{H}, r)$ has the the form

$$
k_{\mathcal{B}(\mathcal{H}, r) ; \lambda}=\frac{1}{\pi} \frac{\Theta_{\mathcal{H}}^{+}(r, z) \Theta_{\mathcal{H}}^{-}(r, \bar{\lambda})-\Theta_{\mathcal{H}}^{-}(r, z) \Theta_{\mathcal{H}}^{+}(r, \bar{\lambda})}{z-\bar{\lambda}}, \quad z \in \mathbb{C} .
$$


The Paley-Wiener space $\mathrm{PW}_{r}$ is the de Branges space $\mathcal{B}\left(\mathcal{H}_{0}, r\right)$ for the Hamiltonian $\mathcal{H}_{0}=\left(\begin{array}{ll}1 & 0 \\ 0 & 1\end{array}\right)$. The reproducing kernel of $\mathrm{PW}_{r}$ at $\lambda \in \mathbb{C}$ will be denoted by sinc ${ }_{r, \lambda}$ :

$$
\operatorname{sinc}_{r, \lambda}=\frac{\sin r(z-\bar{\lambda})}{\pi(z-\bar{\lambda})}, \quad z \in \mathbb{C}
$$

Using integration by parts and equation (2), it is easy to show that for each $\lambda \in \mathbb{C}$ we have

$$
\mathcal{W}_{\mathcal{H}, r} \Theta_{\mathcal{H}}(\cdot, \bar{\lambda})=\sqrt{\pi} k_{\mathcal{B}(\mathcal{H}, r) ; \lambda}, \quad \mathcal{W}_{\mathcal{H}_{0}, r} \Theta_{\mathcal{H}_{0}}(\cdot, \bar{\lambda})=\sqrt{\pi} \operatorname{sinc}_{r, \lambda},
$$

where $\Theta_{\mathcal{H}}(\cdot, \bar{\lambda})$ denotes the mapping $t \mapsto \Theta_{\mathcal{H}}(t, \bar{\lambda})$ and $\Theta_{\mathcal{H}_{0}}(\cdot, \bar{\lambda})$ is defined analogously.

Next assertion is Lemma 4.2 in [2].

Lemma 3.4. Let $\mu$ be a sampling measure for $\mathrm{PW}_{a}$ and let $r \in[0, a]$. The reproducing kernel of the space $\left(\mathrm{PW}_{r}, \mu\right)$ at $\lambda \in \mathbb{C}$ equals $T_{\mu, r}^{-1}$ sinc $_{r, \lambda}$.

Proof. For every function $f$ in $\left(\mathrm{PW}_{r}, \mu\right) \subset \mathrm{PW}_{r}$ and every $\lambda \in \mathbb{C}$ we have

$$
f(\lambda)=\left(f, \operatorname{sinc}_{a, \lambda}\right)_{L^{2}(\mathbb{R})}=\left(f, T_{\mu, r}^{-1} \operatorname{sinc}_{r, \lambda}\right)_{L^{2}(\mu)},
$$

where we used the fact that $c_{1} I \leqslant T_{\mu, a} \leqslant c_{2} I$ on $\mathrm{PW}_{a}$ and hence $T_{\mu, r}$ is bounded and invertible on $\mathrm{PW}_{r}$.

Lemma 3.5. Let $\varphi$ be a function on $[0, a]$ such that $e^{|\varphi|} \in L^{1}[0, a]$. Assume that a spectral measure $\mu$ of problem (2) for the canonical Hamiltonian system generated by $\mathcal{H}=\left(\begin{array}{cc}e^{\varphi} & 0 \\ 0 & e^{-\varphi}\end{array}\right)$ satisfies (11) for some constants $c_{1}, c_{2}$. Then function $w=e^{\varphi}$ belongs to the Muckenhoupt class $A_{2}[0, a]$ and $\|w\|_{A_{2}[0,2]} \leqslant 2^{28} c^{14}$, where $c=c_{1}^{-1}+c_{2}^{2} c_{1}^{-1}$. We also have $\frac{1}{a} \int_{0}^{a}\left(w+\frac{1}{w}\right) d x \leqslant 4 c$.

Proof. Let us obtain estimates (6), (7) for the function $\varphi$ as it was suggested in Proposition 3.2 of [2]. Take $r \in[0, a]$. Set $\mathcal{H}_{0}=\left(\begin{array}{ll}1 & 0 \\ 0 & 1\end{array}\right)$ and consider the corresponding Weyl-Titchmarsch transforms

$$
\mathcal{W}_{\mathcal{H}_{0}, r}: L^{2}\left(\mathcal{H}_{0}, r\right) \rightarrow \mathcal{B}\left(\mathcal{H}_{0}, r\right), \quad \mathcal{W}_{\mathcal{H}, r}: L^{2}(\mathcal{H}, r) \rightarrow \mathcal{B}(\mathcal{H}, r) .
$$

We have $\mathcal{B}\left(\mathcal{H}_{0}, r\right)=\mathrm{PW}_{r}$ and $\mathcal{B}(\mathcal{H}, r)=\left(\mathrm{PW}_{r}, \mu\right)$, see Lemma 3.3. Since $\mu$ satisfies (11), the spaces $\mathrm{PW}_{r},\left(\mathrm{PW}_{r}, \mu\right)$ coincide as sets and

$$
c_{2}^{-1}\|f\|_{L^{2}(\mathbb{R})}^{2} \leqslant\left\|T_{\mu, r}^{-1} f\right\|_{L^{2}(\mu)}^{2} \leqslant c_{1}^{-1}\|f\|_{L^{2}(\mathbb{R})}^{2}
$$

for every function $f \in \mathrm{PW}_{r}$. Hence, the operator $T=\mathcal{W}_{\mathcal{H}, r}^{-1} T_{\mu, r}^{-1} \mathcal{W}_{\mathcal{H}_{0}, r}$ from $L^{2}\left(\mathcal{H}_{0}, r\right)$ to $L^{2}(\mathcal{H}, r)$ is correctly defined, bounded, and invertible. Moreover,

$$
c_{2}^{-1}\|X\|_{L^{2}\left(\mathcal{H}_{0}, r\right)}^{2} \leqslant\|T X\|_{L^{2}(\mathcal{H}, r)}^{2} \leqslant c_{1}^{-1}\|X\|_{L^{2}\left(\mathcal{H}_{0}, r\right)}^{2}
$$

for every $X \in L^{2}\left(\mathcal{H}_{0}\right)$. Next, by Lemma 3.4 for each $z \in \mathbb{C}$ we have

$$
T \Theta_{\mathcal{H}_{0}}(\cdot, z)=\mathcal{W}_{\mathcal{H}, r}^{-1}\left(\sqrt{\pi} T_{\mu, r}^{-1} \operatorname{sinc}_{r, \bar{z}}\right)=\Theta_{\mathcal{H}}(\cdot, z) .
$$

For $z=0$ and all $t \in[0, r]$ we have $\Theta_{\mathcal{H}}(t, 0)=\Theta_{\mathcal{H}_{0}}(t, 0)=\left(\begin{array}{l}1 \\ 0\end{array}\right)$, hence

$$
c_{2}^{-1}\left\|\left(\begin{array}{l}
1 \\
0
\end{array}\right)\right\|_{L^{2}\left(\mathcal{H}_{0}, r\right)}^{2} \leqslant\left\|\left(\begin{array}{l}
1 \\
0
\end{array}\right)\right\|_{L^{2}(\mathcal{H}, r)}^{2} \leqslant c_{1}^{-1}\left\|\left(\begin{array}{l}
1 \\
0
\end{array}\right)\right\|_{L^{2}\left(\mathcal{H}_{0}, r\right)}^{2} .
$$

This relation is inequality (7) for the function $\varphi$ and constants $b_{1}=c_{2}^{-1}, b_{2}=c_{1}^{-1}$. 
Now let $\partial_{0}^{n} \Theta_{\mathcal{H}}(\cdot, 0)$ denote the derivative of order $n$ of the mapping $z \mapsto \Theta_{\mathcal{H}}(\cdot, z)$ from $\mathbb{C}$ to $L^{2}(\mathcal{H}, r)$ at the point $z=0$. Then $T \partial_{0}^{n} \Theta_{\mathcal{H}}(\cdot, 0)=\partial_{0}^{n} \Theta_{\mathcal{H}_{0}}(\cdot, 0)$ for all integers $n \geqslant 1$. The right inequality in (19) yields

$$
\left\|\partial_{0}^{n} \Theta_{\mathcal{H}}(\cdot, 0)\right\|_{L^{2}(\mathcal{H}, r)}^{2} \leqslant c_{1}^{-1}\left\|\partial_{0}^{n} \Theta_{\mathcal{H}_{0}}(\cdot, 0)\right\|_{L^{2}\left(\mathcal{H}_{0}, r\right)}^{2} .
$$

From equation (2) we obtain

$$
\begin{gathered}
\partial_{0}^{n} \Theta_{\mathcal{H}}(t, 0)=n ! \int_{0}^{t} \int_{0}^{t_{1}} \ldots \int_{0}^{t_{n-1}} J^{*} \mathcal{H}\left(t_{1}\right) J^{*} \mathcal{H}\left(t_{2}\right) \ldots J^{*} \mathcal{H}\left(t_{n}\right)\left(\begin{array}{l}
1 \\
0
\end{array}\right) d t_{n} \ldots d t_{1} \\
\partial_{0}^{n} \Theta_{\mathcal{H}_{0}}(t, 0)=J^{* n}\left(\begin{array}{c}
t^{n} \\
0
\end{array}\right)
\end{gathered}
$$

for all $t \in[0, r]$ and $n \geqslant 1$. Observe that

$$
J^{*} \mathcal{H}\left(t_{1}\right) J^{*} \mathcal{H}\left(t_{2}\right) \ldots J^{*} \mathcal{H}\left(t_{n}\right)\left(\begin{array}{l}
1 \\
0
\end{array}\right)=\left\{\begin{array}{cc}
\left(\begin{array}{c}
(-1)^{\frac{n+3}{2}} \exp \left(G_{\varphi, n}(t)\right)
\end{array}\right), & n \text { is odd }, \\
\left((-1)^{\frac{n}{2}} \underset{\exp \left(G_{\varphi, n}(t)\right)}{0}\right), & n \text { is even },
\end{array}\right.
$$

where $t=\left(t_{1}, \ldots, t_{n}\right)$ is a point in simplex $K_{t, n}$, and $G_{\varphi, n}$ is defined on $K_{t, n}$ by formula (5). Substitute this representation of $J^{*} \mathcal{H}\left(t_{1}\right) J^{*} \mathcal{H}\left(t_{2}\right) \ldots J^{*} \mathcal{H}\left(t_{n}\right)\left(\begin{array}{l}1 \\ 0\end{array}\right)$ to (21). Then (21), (22), and (20) give us inequality (6) for all $n \geqslant 1$ and all $r \in[0, a]$. It remains to use Proposition $\left[2.1\right.$ to see that $w \in A_{2}[0, a]$ and $\|w\|_{A_{2}[0, a]} \leqslant 2^{28} c^{14}$. The estimate $\frac{1}{a} \int_{0}^{a}\left(w+\frac{1}{w}\right) d x \leqslant 4 c$ follows from Lemma 2.1

3.3. Proof of Theorem 1, Let $\mu$ be a measure on $\mathbb{R}$ such that estimate (1) holds for some $a>0$. Consider the truncated Toeplitz operator $T_{\mu, a}=T_{\mu}$ on $\mathrm{PW}_{a}$. We have $c_{1} I \leqslant T_{\mu} \leqslant c_{2} I$, where $I$ stands for the identity operator on $\mathrm{PW}_{a}$. The operator $T_{\mu}-c_{1} I$ satisfies assumptions of Lemma 3.1. Hence, there exists a measure $\nu \geqslant 0$ on $\mathbb{R}$ such that $T_{\nu}=T_{\mu}-c_{1} I$. One can suppose that $\nu$ is even (otherwise consider the measure $\tilde{\nu}$ such that $\tilde{\nu}(S)=\frac{1}{2}(\nu(S)+\nu(-S))$, and note that $\left.T_{\nu}=T_{\tilde{\nu}}\right)$. Define a sequence of measures $\mu_{j}$ by $\mu_{j}=c_{1} m+\chi_{j} \nu$, where $m$ is the Lebesgue measure on $\mathbb{R}$, and $\chi_{j}$ denotes the indicator function of the interval $[-j, j]$. For every $j \geqslant 1$ the measure $\mu_{j}$ is even and satisfies relation (11) with the same constants $c_{1}, c_{2}$. Indeed, $T_{\mu_{j}}=c_{1} I+T_{\chi_{j} \nu}$ and

$$
c_{1} I \leqslant c_{1} I+T_{\chi_{j} \nu} \leqslant c_{1} I+T_{\nu}=T_{\mu} \leqslant c_{2} I .
$$

By Lemma 3.2 and Lemma 3.5, for every $j$ there exists a smooth function $w_{j}>0$ on the interval $[0, a]$ such that $\left\|w_{j}\right\|_{A_{2}[0, a]} \leqslant 2^{28} c^{14}, c=c_{1}^{-1}+c_{2}^{2} c_{1}^{-1}$, and $\mu_{j}$ is the spectral measure for the Hamiltonian $\mathcal{H}_{j}=\left(\begin{array}{cc}w_{j} & 0 \\ 0 & \frac{1}{w_{j}}\end{array}\right)$ on $[0, a]$. We also have $\frac{1}{a} \int_{0}^{a}\left(w+\frac{1}{w}\right) d x \leqslant 4 c$ for all $j \geqslant 1$. This allows us to use "a reverse Hölder inequality" for weights in $A_{2}[0, a]$. It says that for every $C_{1}>0$ there exist $p>1$ and $C_{2}>0$ such that for all $h \in A_{2}[0, a]$ with $\|h\|_{A_{2}[0, a]} \leqslant C_{1}$ we have

$$
\frac{1}{a} \int_{0}^{a} h(x)^{p} d x \leqslant C_{2}\left(\frac{1}{a} \int_{0}^{a} h(x) d x\right)^{p} .
$$

Explicit relations between $C_{1}, C_{2}$, and $p$ can be found in [19]. From here we see that sequences $\left\{w_{j}\right\}_{j \geqslant 1},\left\{\frac{1}{w_{j}}\right\}_{j \geqslant 1}$ are informly bounded in $L^{p}[0, a]$ for some $p>1$. Hence we can find subsequences $w_{j_{k}}, w_{j_{k}}^{-1}$ converging weakly in $L^{p}[0, a]$ to functions $w, v$, correspondingly. To simplify notations, let the sequences $\left\{w_{j}\right\}_{j} \geqslant 1,\left\{\frac{1}{w_{j}}\right\}_{j \geqslant 1}$ themselves be weakly convergent. Let us show that $v=w^{-1}$ almost everywhere 
on the interval $[0, a]$. This is not always the case for arbitrary weakly convergent sequences in $L^{p}[0, a]$.

For $z \in \mathbb{C}$ denote by $\Theta_{j}(\cdot, z)$ solution of equation (2) for the Hamiltonian $\mathcal{H}_{j}$. Integrating (2), we get

$$
J \Theta_{j}(r, z)-\left(\begin{array}{l}
1 \\
0
\end{array}\right)=z \int_{0}^{r} \mathcal{H}_{j}(t) \Theta_{j}(t, z) d t .
$$

Then for every $j \geqslant 1$ and $r, r^{\prime} \in[0, a]$ we have the estimates

$$
\begin{gathered}
\left\|\Theta_{j}(r, z)\right\|_{\mathbb{C}^{2}} \leqslant \exp \left(|z| \int_{0}^{a}\left\|\mathcal{H}_{j}(t)\right\| d t\right) \\
\left\|\Theta_{j}(r, z)-\Theta_{j}\left(r^{\prime}, z\right)\right\|_{\mathbb{C}^{2}} \leqslant|z| \cdot\left|r-r^{\prime}\right|^{\frac{p-1}{p}}\left(\int_{0}^{a}\left\|\mathcal{H}_{j}(t)\right\|^{p} \cdot\left\|\Theta_{j}(t, z)\right\|_{\mathbb{C}^{2}}^{p} d t\right)^{\frac{1}{p}}
\end{gathered}
$$

showing that functions $\Theta_{j}(\cdot, z)$ are uniformly bounded and equicontinuous on $[0, a]$. Therefore, there is a subsequence of the sequence $\Theta_{j}(\cdot, z)$ converging uniformly on $[0, a]$ to a function $\Theta(\cdot, z)$. As before, we suppose that the sequence $\Theta_{j}(\cdot, z)$ itself is uniformly convergent on $[0, a]$. It is clear that the limit function $\Theta$ satisfies equation (23) for the Hamiltonian $\mathcal{H}=\left(\begin{array}{cc}w & 0 \\ 0 & v\end{array}\right)$. Hence, it satisfies equation (2) for $\mathcal{H}$. Fix a number $r \in(0, a]$. For every $\lambda$ and $z$ in $\mathbb{C}$ we have

$$
k_{\mathcal{B}(\mathcal{H}, r) ; \lambda}(z)=\lim _{j \rightarrow \infty} k_{\mathcal{B}\left(\mathcal{H}_{j}, r\right) ; \lambda}(z)=\lim _{j \rightarrow \infty}\left(T_{\mu_{j}, r}^{-1} \operatorname{sinc}_{r, \lambda}\right)(z)=\left(T_{\mu, r}^{-1} \operatorname{sinc}_{r, \lambda}\right)(z) .
$$

Indeed, the first equality above follows from formula (18) and convergence of $\Theta_{j}$ to $\Theta$ on $[0, a]$ when a spectral parameter $(\bar{\lambda}$ or $z)$ is fixed. Lemma 3.3 and Lemma 3.4 give us the second equality. Finally, using the fact that the operators $T_{\mu_{j}, r}$ on $\mathrm{PW}_{r}$ tend to $T_{\mu, r}$ in the strong operator topology, we obtain the last equality in (24). From (24) we see that Hilbert spaces of entire functions $\mathcal{B}(\mathcal{H}, r),\left(\mathrm{PW}_{r}, \mu\right)$ have the same reproducing kernels. Hence $\mathcal{B}(\mathcal{H}, r)=\left(\mathrm{PW}_{r}, \mu\right)$ and formula (17) implies

$$
r=\int_{0}^{r} \sqrt{\operatorname{det} \mathcal{H}(t)} d t, \quad r \in[0, a] .
$$

It follows that $\operatorname{det} \mathcal{H}=1$ almost everywhere on $[0, a]$, that is, $v=w^{-1}$. Next, from the direct spectral theory we know that the family $\{\Theta(\cdot, \lambda)\}_{\lambda \in \mathbb{C}}$ is complete in $L^{2}(\mathcal{H}, a)$ and $\mathcal{W}_{\mathcal{H}, a} \Theta(\cdot, \lambda)=k_{\mathcal{B}(\mathcal{H}, a) ; \lambda}$ for every $\lambda \in \mathbb{C}$, where $\mathcal{W}_{\mathcal{H}, a}$ denotes the Weyl-Titchmarsch transform associated to $\mathcal{H}$. Using (24) again, we get

$$
\begin{aligned}
& (\Theta(\cdot, \lambda), \Theta(\cdot, z))_{L^{2}(\mathcal{H}, a)}=\pi k_{\mathcal{B}(\mathcal{H}, a) ; \lambda}(z)=\pi\left(T_{\mu, a}^{-1} \operatorname{sinc}_{a, \lambda}, \operatorname{sinc}_{a, z}\right)_{L^{2}(\mathbb{R})} \\
& =\pi\left(T_{\mu, a}^{-1} \operatorname{sinc}_{a, \lambda}, T_{\mu, a}^{-1} \operatorname{sinc}_{a, z}\right)_{L^{2}(\mu)}=\left(\mathcal{W}_{\mathcal{H}, a} \Theta(\cdot, \lambda), \mathcal{W}_{\mathcal{H}, a} \Theta(\cdot, z)\right)_{L^{2}(\mu)} .
\end{aligned}
$$

Hence, the operator $\mathcal{W}_{\mathcal{H}, a}$ acts isometrically from $L^{2}(\mathcal{H} ; a)$ to $L^{2}(\mu)$ and $\mu$ is a spectral measure for $\mathcal{H}$. In particular, we can apply Lemma 3.5 to $\mathcal{H}, \mu$, and conclude that the function $w=e^{\varphi}$ is in $A_{2}[0, a]$ and $\|w\|_{A_{2}[0, a]} \leqslant 2^{28} c^{14}$. Uniqueness of the Hamiltonian $\mathcal{H}$ follows immediately from formula (24):

$$
\int_{0}^{r} w(t) d t=\int_{0}^{r}\left\langle\mathcal{H}(t)\left(\begin{array}{l}
1 \\
0
\end{array}\right),\left(\begin{array}{l}
1 \\
0
\end{array}\right)\right\rangle d t=\pi k_{\mathcal{B}(\mathcal{H}, a) ; 0}(0)=\pi\left\|T_{\mu, r}^{-1} \operatorname{sinc}_{r, 0}\right\|_{L^{2}(\mu)}^{2},
$$

where the right hand side is completely determined by $\mu$, while the left hand side determines $\mathcal{H}$. 
Differentiating formula (25), we obtain the following corollary.

Corollary 1. The Hamiltonian $\mathcal{H}=\left(\begin{array}{cc}w & 0 \\ 0 & \frac{1}{w}\end{array}\right)$ in Theorem 1 could be recovered from $\mu$ by means of the following formula: $w(r)=\pi \frac{\partial}{\partial r}\left\|T_{\mu, r}^{-1} \operatorname{sinc}_{r, 0}\right\|_{L^{2}(\mu)}^{2}, r \in[0, a]$.

\section{Proof of Theorem 2 and Theorem 3}

Let us first show that Theorem 2 does not follow from a general theory of canonical Hamiltonian systems. Consider the simplest case where the Hamiltonian $\mathcal{H}$ coincides with the identity matrix $\left(\begin{array}{ll}1 & 0 \\ 0 & 1\end{array}\right)$ on $[0, a]$. We claim that there is no unitary operator $U: L^{2}(\mathcal{H}, a) \rightarrow \mathrm{PW}_{[0,2 a]}$ such that $U L^{2}(\mathcal{H}, r)=\mathrm{PW}_{[0,2 r]}$ for all $r \in[0, a]$. Indeed, existence of such a unitary operator yields the existence of another unitary operator $\tilde{U}: L^{2}[-a, a] \rightarrow L^{2}[0,2 a]$ such that $\tilde{U} L^{2}[-r, r]=L^{2}[0,2 r]$ for all $r \in[0, a]$. For every $r_{1}>r_{2} \geqslant 0$ let $\chi_{\left[r_{1}, r_{2}\right]}$ denote the indicator function of the interval $\left[r_{1}, r_{2}\right]$. Put $g=\tilde{U} \chi_{[0, a]}$ and consider decomposition $g=f_{r}+h_{r}$, where $f_{r}=\tilde{U} \chi_{[0, r]}, h_{r}=\tilde{U} \chi_{[r, a]}, r \in[0, a]$. Since $\tilde{U} L^{2}[-r, r]=L^{2}[0,2 r]$ by our assumption, the function $f_{r}$ is supported on $[0,2 r]$. Note also that the function $h_{r}$ is orthogonal to all functions from $L^{2}[0,2 r]$ and hence it is supported on $[2 r, 2 a]$. From here we see that $f_{r}=\chi_{[0,2 r]} g$ for all $r \in[0, a]$. Next, unitarity of the operator $\tilde{U}$ implies that

$$
\int_{0}^{2 r}|g(t)|^{2} d t=\int_{0}^{2 r}\left|f_{r}(t)\right|^{2} d t=\int_{-a}^{a}\left|\chi_{[0, r]}(t)\right|^{2} d t=r, \quad r \in[0, a] .
$$

It follows that $|g(t)|^{2}=1 / 2$ for almost all $t \in[0,2 a]$. In particular, the linear span of functions $f_{r} \in \tilde{U} L^{2}[0, a], r \in[0, a]$, is dense in $L^{2}[0,2 a]$. This contradicts to the fact that $\tilde{U}$ is a unitary operator from $L^{2}[-a, a]$ to $L^{2}[0,2 a]$. Thus, the WeylTitchmarsh transform $\mathcal{W}_{\mathcal{H}, a}$ from formula (3) can not be used to construct the operator $\mathcal{F}_{\mu}$ from Theorem 2 by means of superpositon with some simple unitary operators like shifts, reflections, etc.

The main point that helps in proof of Theorem 2 is the fact that Hamiltonian $\mathcal{H}$ generated by an even sampling measure for the Paley-Wiener space $\mathrm{PW}_{a}$ must have rank two almost everywhere on its domain of definition. It is an open question if this is true for general (not necessarily even) sampling measures for $\mathrm{PW}_{a}$. See also Proposition 5.1 in Section 5 for more details.

Proof of Theorem 2, Fix an even sampling measure $\mu$ and construct the Hamiltonians $\mathcal{H}_{j}, \mathcal{H}$, on $[0, a]$ as in the proof of Theorem[1. Put $\varphi_{j}=\log w_{j}$ and $\varphi=\log w$, where $w_{j}, w$ are the functions generating $\mathcal{H}_{j}, \mathcal{H}$. Recall that $w_{j}$ tend to $w$ weakly in $L^{p}[0, a]$ for some $p>1$ and the same is true for $w_{j}^{-1}$ and $w^{-1}$. Let $\Theta_{j}, \Theta$ be the solutions of system (2) generated by Hamiltonians $\mathcal{H}_{j}, \mathcal{H}$, correspondingly. As we have seen, the functions $\Theta_{j}(\cdot, z)=\left(\begin{array}{c}\Theta_{j}^{+} \\ \Theta_{j}^{-}\end{array}\right)$converge uniformly to $\Theta(\cdot, z)=\left(\begin{array}{c}\Theta^{+} \\ \Theta^{-}\end{array}\right)$ on the interval $[0, a]$ when $z \in \mathbb{C}$ is fixed. For $r \in[0, a]$, define entire functions $P_{2 r, j}$ 
and $P_{2 r, j}^{*}$ by

$$
\begin{aligned}
& P_{2 r, j}: z \mapsto e^{i r z}\left(e^{\frac{\varphi_{j}(r)}{2}} \Theta_{j}^{+}(r, z)-i e^{-\frac{\varphi_{j}(r)}{2}} \Theta_{j}^{-}(r, z)\right), \\
& P_{2 r, j}^{*}: z \mapsto e^{i r z}\left(e^{\frac{\varphi_{j}(r)}{2}} \Theta_{j}^{+}(r, z)+i e^{-\frac{\varphi_{j}(r)}{2}} \Theta_{j}^{-}(r, z)\right),
\end{aligned}
$$

and let $P_{2 r}, P_{2 r}^{*}$ be defined similarly with $\varphi_{j}$ replaced by $\varphi$. These functions satisfy the Krein system of differential equations:

$$
\begin{cases}P_{r, j}^{\prime}(z)=i z P_{r, j}(z)+\frac{\varphi_{j}^{\prime}(r / 2)}{4} P_{r, j}^{*}(z), & P_{0, j}(z)=e^{\frac{\varphi_{j}(0)}{2}}, \\ P_{r, j}^{* \prime}(z)=\frac{\varphi_{j}^{\prime}(r / 2)}{4} P_{r, j}(z), & P_{0, j}^{*}(z)=e^{\frac{\varphi_{j}(0)}{2}},\end{cases}
$$

where $\varphi_{j}^{\prime}(r / 2)$ is the value of smooth function $\varphi_{j}^{\prime}$ at $r / 2$. From system (26) we obtain by integration by parts (see Lemma 9.1 in [5]) the Christoffel-Darboux formula:

$$
\int_{0}^{r} P_{t, j}(z) \overline{P_{t, j}(\lambda)} d t=i \frac{P_{r, j}^{*}(z) \overline{P_{r, j}^{*}(\lambda)}-P_{r, j}(z) \overline{P_{r, j}(\lambda)}}{z-\bar{\lambda}} .
$$

The right hand side could be rewritten in the form

$$
\ldots=2 e^{i \frac{r}{2}(z-\bar{\lambda})} \cdot \frac{\Theta_{j}^{+}\left(\frac{r}{2}, z\right) \overline{\Theta_{j}^{-}\left(\frac{r}{2}, \lambda\right)}-\Theta_{j}^{-}\left(\frac{r}{2}, z\right) \overline{\Theta_{j}^{+}\left(\frac{r}{2}, \lambda\right)}}{z-\bar{\lambda}},
$$

which tends to $2 \pi k_{r, \lambda}(z)$, the scalar multiple of the reproducing kernel $k_{r, \lambda}$ at $\lambda$ of the Hilbert space $e^{i \frac{r}{2} z} \mathcal{B}\left(\mathcal{H}, \frac{r}{2}\right)=\left(\mathrm{PW}_{[0, r]}, \mu\right)$, see formula (18). On the other hand, for every pair $z, \lambda \in \mathbb{C}$ we have

$$
\begin{aligned}
P_{t, j}(z) \overline{P_{t, j}(\lambda)}=e^{i \frac{t}{2}(z-\bar{\lambda})}\left(e^{\varphi_{j}\left(\frac{t}{2}\right)} \Theta_{j}^{+}\left(\frac{t}{2}, z\right) \overline{\Theta_{j}^{+}\left(\frac{t}{2}, \lambda\right)}+e^{-\varphi_{j}\left(\frac{t}{2}\right)} \Theta_{j}^{-}\left(\frac{t}{2}, z\right) \overline{\Theta_{j}^{-}\left(\frac{t}{2}, \lambda\right)}+\right. \\
\left.+i \Theta_{j}^{+}\left(\frac{t}{2}, z\right) \overline{\Theta_{j}^{-}\left(\frac{t}{2}, \lambda\right)}-i \Theta_{j}^{-}\left(\frac{t}{2}, z\right) \overline{\Theta_{j}^{+}\left(\frac{t}{2}, \lambda\right)}\right) .
\end{aligned}
$$

Since functions $e^{\varphi_{j}}, e^{-\varphi_{j}}$ converge weakly in $L^{p}[0, a]$ to functions $e^{\varphi}, e^{-\varphi}$, correspondingly, we see that

$$
\int_{0}^{r} P_{t}(z) \overline{P_{t}(\lambda)} d t=\lim _{j \rightarrow \infty} \int_{0}^{r} P_{t, j}(z) \overline{P_{t, j}(\lambda)} d t=2 \pi k_{r, \lambda}(z)
$$

for every $r \in[0,2 a]$. Let $\chi_{r}$ be the indicator function of the interval $[0, r]$. Denote by $\mathrm{L}$ the set of all finite linear combinations of functions $t \mapsto \chi_{r}(t) \overline{P_{t}(z)}$ on $[0,2 a]$, where $z \in \mathbb{C}$ and $r \in[0,2 a]$. The linear manifold $\mathrm{L}$ is dense in $L^{2}[0,2 a]$. Indeed, for every function $g \in L^{2}[0,2 a]$ orthogonal to $\mathrm{L}$ we have

$$
0=\int_{0}^{2 a} g(t) \chi_{r}(t) P_{t}(0) d t=\int_{0}^{r} g(t) e^{\frac{\varphi(t / 2)}{2}} d t, \quad r \in[0,2 a],
$$

yielding $g=0$ in $L^{2}[0,2 a]$. Formula (27) also shows that a nontrivial finite linear combination of functions $\chi_{r}(t) \overline{P_{t}(z)}$ cannot vanish almost everywhere on $[0,2 a]$. Consider the operator $\mathcal{F}_{\mu}: L^{2}[0,2 a] \rightarrow\left(\mathrm{PW}_{[0,2 a]}, \mu\right)$ densely defined on $\mathrm{L}$ by

$$
\mathcal{F}_{\mu}: f \mapsto \frac{1}{\sqrt{2 \pi}} \int_{0}^{2 a} f(t) P_{t}(z) d t, \quad z \in \mathbb{C} .
$$


The operator $\mathcal{F}_{\mu}$ takes the function $t \mapsto \chi_{r}(t) \overline{P_{t}(\lambda)}$ on $[0,2 a]$ into $\sqrt{2 \pi} k_{r, \lambda}$, see formula (27). Moreover, for every $r_{1}, r_{2} \in[0,2 a]$ we have

$$
\begin{aligned}
\left(\mathcal{F}_{\mu} \chi_{r_{1}} \overline{P_{t}(\lambda)}, \mathcal{F}_{\mu} \chi_{r_{2}} \overline{P_{t}(z)}\right)_{L^{2}(\mu)} & =2 \pi\left(k_{r_{1}, \lambda}, k_{r_{2}, z}\right)_{L^{2}(\mu)}=2 \pi k_{r, \lambda}(z), \\
& =\left(\chi_{r_{1}} \overline{P_{t}(\lambda)}, \chi_{r_{2}} \overline{P_{t}(z)}\right)_{L^{2}[0,2 a]},
\end{aligned}
$$

where $r=\min \left(r_{1}, r_{2}\right)$. This shows that $\mathcal{F}_{\mu}$ is an isometry on L. Since the linear span of the set $\left\{k_{2 a, \lambda}, \lambda \in \mathbb{C}\right\}$ is complete in $\left(\mathrm{PW}_{[0,2 a]}, \mu\right)$, the operator $\mathcal{F}_{\mu}$ is unitary. It is also clear from the definition that $\mathcal{F}_{\mu}$ maps $L^{2}[0, r]$ onto $\left(\mathrm{PW}_{[0, r]}, \mu\right)$ for every $r \in[0,2 a]$.

Proof of Theorem [3. At first, consider a positive bounded invertible operator $W_{\psi}$ with real symbol $\psi \in \mathcal{S}^{\prime}$ on a finite interval $[0, a]$. Let $\mathcal{F}$ denote the unitary Fourier transform on $L^{2}(\mathbb{R})$. Take a smooth function $h$ with support in $(0, a)$ and put $\hat{f}=\mathcal{F} f$. Consider the operator $\hat{W}_{\psi}=\mathcal{F} W_{\psi} \mathcal{F}^{-1}$ on $\mathrm{PW}_{[0, a]}$. We have $\left(\hat{W}_{\psi} \hat{h}, \hat{h}\right)_{L^{2}(\mathbb{R})}=\left\langle\hat{\psi},|\hat{h}|^{2}\right\rangle_{\mathcal{S}^{\prime}}$, where $\hat{\psi}$ is the Fourier transform of the tempered distribution $\psi$. It follows that

$$
\left(\hat{W}_{\psi} f, f\right)_{L^{2}(\mathbb{R})}=\left(\hat{W}_{\psi} \frac{z-i}{z+i} f, \frac{z-i}{z+i} f\right)_{L^{2}(\mathbb{R})}
$$

on a dense subset of the set $Z_{-i}=\left\{f \in \mathrm{PW}_{[0, a]}: f(-i)=0\right\}$. Since $\hat{W}_{\psi}$ is bounded on $\mathrm{PW}_{[0, a]}$, we have the last identity for all $f \in Z_{-i}$. Hence, the operator $\hat{W}_{\psi}$ satisfies assumptions of Lemma 3.1 and we can find a positive Borel measure $\mu$ on $\mathbb{R}$ such that

$$
\left(\hat{W}_{\psi} f, g\right)_{L^{2}(\mathbb{R})}=\int_{\mathbb{R}} f \bar{g} d \mu
$$

for all $f, g \in \mathrm{PW}_{[0, a]}$. As in the proof of Theorem 10 we can assume that the measure $\mu$ is even. Indeed, since $\psi$ is real, we have $\left(\hat{W}_{\psi} f, f\right)=\left(\hat{W}_{\psi} f^{*}, f^{*}\right)$ for arbitrary $f \in \mathrm{PW}_{[0, a]}$ and its reflection $f^{*}: x \mapsto f(-x)$. By the assumption, the operator $\hat{W}_{\psi}$ is positive, bounded and invertible on $\mathrm{PW}_{[0, a]}$. Hence the measure $\mu$ satisfies (11) for some $c_{1}, c_{2}$ and $a / 2$ in place of $a$. By Theorem 2, there is a unitary operator $\mathcal{F}_{\mu}: L^{2}[0, a] \rightarrow\left(\mathrm{PW}_{[0, a]}, \mu\right)$ such that $\mathcal{F}_{\mu}: L^{2}[0, r]=\left(\mathrm{PW}_{[0, r]}, \mu\right)$ for every $r \in[0, a]$. Identifying Hilbert spaces $\left(\mathrm{PW}_{[0, a]}, \mu\right)$ and $\mathrm{PW}_{[0, a]}$ as sets, we can define the operator $A=\mathcal{F}_{\mu}^{-1} \mathcal{F}$ on $L^{2}[0, a]$. By construction, the operator $A$ is bounded and invertible and $A L^{2}[0, r]=L^{2}[0, r]$ for every $r \in[0, a]$. We also have

$$
\left(W_{\psi} h, h\right)_{L^{2}[0, a]}=\int_{\mathbb{R}}|\hat{h}|^{2} d \mu=(\mathcal{F} h, \mathcal{F} h)_{L^{2}(\mu)}=\left(\mathcal{F}_{\mu}^{-1} \mathcal{F} h, \mathcal{F}_{\mu}^{-1} \mathcal{F} h\right)_{L^{2}[0, a]}
$$

for all smooth functions $h$ with support in $(0, a)$. It follows that the operator $W_{\psi}$ admits the triangular factorization $W_{\psi}=A^{*} A$.

It remains to consider the case where $W_{\psi}$ is a positive bounded invertible WienerHopf operator on $L^{2}[0, \infty)$ with real symbol $\psi \in \mathcal{S}^{\prime}$. It is known (see Section 4.2.7 in [14]) that in this case the Fourier transform of the distribution $\psi$ coincides with a function $\sigma$ on $\mathbb{R}$ such that $c_{1} \leqslant \sigma(x) \leqslant c_{2}$ for some positive constants $c_{1}, c_{2}$ and almost all $x \in \mathbb{R}$. In particular, the measure $\mu=\sigma d m$ is sampling for all Paley-Wiener spaces $\mathrm{PW}_{[0, r]}, r>0$. Since $\psi$ is real, the function $\sigma$ is even. For every $r>1$ we can use Theorem 1 and find a Hamiltonian $\mathcal{H}_{r}$ on $[0, r]$ such that $\operatorname{det} \mathcal{H}_{r}(t)=1$ for almost all $t \in[0, r]$ and $\mu$ is the spectral measure for $\mathcal{H}_{r}$. Since the Hamiltonian $\mathcal{H}$ in Theorem 1 is defined uniquely, we have $\mathcal{H}_{r}(t)=\mathcal{H}_{r^{\prime}}(t)$ for 
almost all $t \in\left[0, \min \left(r, r^{\prime}\right)\right]$. This shows that there is the Hamiltonian $\mathcal{H}$ on $[0, \infty)$ such that $\operatorname{det} \mathcal{H}=1$ almost everywhere and $\mu$ is the spectral measure for $\mathcal{H}$. In particular, we can define a family of entire functions $\left\{P_{t}\right\}_{t \geqslant 0}$ such that the mapping

$$
\mathcal{F}_{\mu}: f \mapsto \frac{1}{\sqrt{2 \pi}} \int_{0}^{r} f(t) P_{t}(z) d t
$$

sends unitarily the space $L^{2}[0, r]$ onto the space $\left(\mathrm{PW}_{[0, r]}, \mu\right)$ for every $r>0$, see the proof of Theorem 2, Let $H_{\mu}^{2}\left(\mathbb{C}_{+}\right)$be the weighted Hardy space with the inner product $(f, g)_{H_{\mu}^{2}\left(\mathbb{C}_{+}\right)}=(f, g)_{L^{2}(\mu)}$. Since $c_{1} \leqslant \sigma \leqslant c_{2}$ on $\mathbb{R}$, the space $H_{\mu}^{2}\left(\mathbb{C}_{+}\right)$ coincides as a set with the standard Hardy space $H^{2}\left(\mathbb{C}_{+}\right)=\mathcal{F} L^{2}[0, \infty)$. Define the unitary operator $\mathcal{F}_{\mu}$ from $L^{2}[0, \infty)$ to $H_{\mu}^{2}\left(\mathbb{C}_{+}\right)$by formula (29) with $r=\infty$ on the dense set of compactly supported bounded functions in $L^{2}[0, \infty)$. Then the operator $A=\mathcal{F}_{\mu}^{-1} \mathcal{F}$ on $L^{2}[0, \infty)$ is bounded and invertible. Moreover, $A L^{2}[0, r]=L^{2}[0, r]$ for every $r \geqslant 0$, and $W_{\psi}=A^{*} A$, see formula (28).

Remark. It can be shown that positive bounded invertible Wiener-Hopf operators $W_{\psi}$ on $L^{2}[0, a)$ with real symbols $\psi \in \mathcal{S}^{\prime}$ admit triangular factorisation in the reverse order, $W_{\psi}=A A^{*}$. In the case $a=\infty$ the classical Wiener-Hopf factorization works: one can take $A=\mathcal{F}^{-1} T_{\bar{\varphi}_{\sigma}} \mathcal{F}$, where $T_{\varphi_{\sigma}}$ is the Toeplitz operator on $H^{2}\left(\mathbb{C}_{+}\right)$ with analytic symbol $\varphi_{\sigma}$ such that $\left|\varphi_{\sigma}\right|^{2}=\sigma=\mathcal{F} \psi$. If $a>0$ is finite, then we can use Theorem 3 to find left triangular factorization $W_{\psi}=\tilde{A}^{*} \tilde{A}$ and then put $A=C_{a} \tilde{A} C_{a}$, where $C_{a}: f \mapsto \overline{f(a-x)}$ is the conjugate-linear isometry on $L^{2}[0, a]$. Since $C_{a} W_{\psi} C_{a}=W_{\psi}$ for the self-adjoint Wiener-Hopf operator $W_{\psi}$ on $L^{2}[0, a]$, and $C_{a}^{2}=I$, we have $W_{\psi}=A A^{*}$. It is also clear that the operator $A$ is upper-triangular.

\section{Appendix. Two Results by L. A. Sakhnovich}

In paper [17] L. A. Sakhnovich proved (see Theorem 4.1 and Remark 4.1 in [17]) that positive bounded invertible Wiener-Hopf operator

$$
T: f \mapsto f-\mu \int_{0}^{\infty} f(t) \frac{\sin \pi(t-x)}{\pi(t-x)} d t, \quad f \in L^{2}[0, \infty), \quad 0<\mu<1,
$$

densely defined on $L^{2}[0, \infty)$ does not admit triangular factorization $T=A^{*} A$, where a bounded invertible operator $A$ on $L^{2}[0, \infty)$ is such that $A L^{2}[0, r]=L^{2}[0, r]$ for every $r \geqslant 0$. Clearly, this assertion contradicts Theorem [3, Let us point out an error in its proof.

The argument in [17] crucially uses the following claim. Let $\chi_{[-\pi, \pi]}$ be the indicator function of the interval $[-\pi, \pi]$. Formulas $(4.1)-(4.4)$ in [17] for $n=0$ and $a_{0}=\pi$ determine the function $\sigma^{\prime}: x \mapsto \frac{1}{2 \pi}\left(1-\mu \cdot \chi_{[-\pi, \pi]}(x)\right)$ on $\mathbb{R}$. The function

$$
\Pi(z)=\frac{1}{\sqrt{2 \pi}} \exp \left(\frac{1}{2 i \pi} \int_{-\infty}^{\infty} \frac{1+t z}{(z-t)\left(1+t^{2}\right)} \log \sigma^{\prime}(t) d t\right)
$$

from formula (4.10) of [17] (see also formula (4.12) therein) is claimed to satisfy the following relation (formula (4.18) in [17]):

$$
\lim _{y \rightarrow+0} \Pi(i y)=\sqrt{1-\mu} .
$$

However, this fact is false. Indeed, we have

$$
\frac{1}{\pi i} \frac{1+t z}{(z-t)\left(1+t^{2}\right)}=-\frac{1}{\pi i}\left(\frac{1}{t-z}-\frac{t}{1+t^{2}}\right)
$$


and hence $\sqrt{2 \pi} \Pi(z)$ is the outer function in $\mathbb{C}_{+}$whose absolute value on $\mathbb{R}$ coincides with $\left(\sigma^{\prime}\right)^{-1 / 2}$ almost everywhere on $\mathbb{R}$. Since $\left(\sigma^{\prime}\right)^{-1 / 2}$ is regular (in fact, constant) near the origin, we have

$$
\lim _{y \rightarrow+0} \Pi(i y)=\frac{1}{\sqrt{2 \pi}}\left(\sigma^{\prime}\right)^{-1 / 2}(0)=\frac{1}{\sqrt{1-\mu}} .
$$

We also would like to note that the last relation agrees well with the first identity in formula (4.19) from [17].

The second part of this section concerns factorization problem for truncated Toeplitz operators generated by general sampling measures for the space $\mathrm{PW}_{a}$ not necessarily symmetric with respect to the origin. The result is equivalent to Theorem 4.2 in [16]. The proof below seems to be a bit more straightforward than the original one, possibly, because we consider the one-dimensional situation.

Proposition 5.1. Let $\mathcal{H}$ be a Hamiltonian on $[0, \ell]$ such that $\int_{0}^{\ell} \operatorname{trace} \mathcal{H}(r)<\infty$, and let $\mu$ be a spectral measure for problem (2). Set $a=\int_{0}^{\ell} \sqrt{\operatorname{det} \mathcal{H}(r)} d r$. Assume that $\mu$ satisfies (1). The following assertions are equivalent:

(a) $\operatorname{det} \mathcal{H}>0$ almost everywhere on $[0, \ell]$;

(b) there exists a unitary operator $V_{\mu}: \mathrm{PW}_{a} \rightarrow\left(\mathrm{PW}_{a}, \mu\right)$ such that for every $r \in[0, a]$ we have $V_{\mu} \mathrm{PW}_{r}=\left(\mathrm{PW}_{a}, \mu\right)$.

(c) there exists a bounded invertible operator $A$ on $\mathrm{PW}_{a}$ such that $T_{\mu, a}=A^{*} A$ and for every $r \in[0, a]$ we have $A \mathrm{PW}_{r}=\mathrm{PW}_{r}$.

Given a Hamiltonian $\mathcal{H}$ on $[0, \ell]$ such that $a=\int_{0}^{\ell} \sqrt{\operatorname{det} \mathcal{H}(t)} d t>0$, we define continuous from the left function $\xi_{\mathcal{H}}$ from $[0, a]$ to $[0, \ell]$ by

$$
r=\int_{0}^{\xi_{\mathcal{H}}(r)} \sqrt{\operatorname{det} \mathcal{H}(t)} d t, \quad r \in[0, a] .
$$

This function is continuous if and only if there are no interval $\left(r_{1}, r_{2}\right) \subset[0, \ell]$ such that $\operatorname{det} \mathcal{H}(t)=0$ for almost all $t \in\left(r_{1}, r_{2}\right)$. The function $\xi_{\mathcal{H}}$ is absolutely continuous if and only if $\operatorname{det} \mathcal{H}(t)>0$ for almost all $t \in[0, a]$, see Exercise 13 in Chapter IX of [13].

Proof of Proposition 5.1. $(a) \Rightarrow(b)$. Since $\operatorname{det} \mathcal{H}>0$ almost everywhere on the interval $[0, \ell]$, the function $\xi=\xi_{\mathcal{H}}$ is absolutely continuous and

$$
\xi^{\prime}(r)=\frac{1}{\sqrt{\operatorname{det} \mathcal{H}(\xi(r))}}
$$

for almost all $r \in[0, a]$. Consider the Hamiltonian $\tilde{\mathcal{H}}: r \mapsto \xi^{\prime}(r) \mathcal{H}(\xi(r))$ on the interval $[0, a]$. We have $\operatorname{det} \tilde{\mathcal{H}}=1$ and $\Theta_{\tilde{\mathcal{H}}}(r, z)=\Theta_{\mathcal{H}}(\xi(r), z)$ on $[0, a]$. Changing variable in (3) $)$, we see that $\mathcal{B}(\tilde{\mathcal{H}}, r)=\mathcal{B}(\mathcal{H}, \xi(r))$ for every $r \in[0, a]$, hence $\mu$ is the spectral measure for $\tilde{\mathcal{H}}$. Consider the Weyl-Titchmarsh transforms generated by Hamiltonians $\tilde{\mathcal{H}}$ and $\mathcal{H}_{0}=\left(\begin{array}{ll}1 & 0 \\ 0 & 1\end{array}\right)$, correspondingly,

$$
\mathcal{W}_{\tilde{\mathcal{H}}, a}: L^{2}(\tilde{\mathcal{H}}, a) \rightarrow \mathcal{B}(\tilde{\mathcal{H}}, a), \quad \mathcal{W}_{\mathcal{H}_{0}, a}: L^{2}\left(\mathcal{H}_{0}, a\right) \rightarrow \mathrm{PW}_{a}
$$

Define the operator $V_{\mu}: \mathrm{PW}_{a} \rightarrow \mathcal{B}(\tilde{\mathcal{H}}, a)$ by $V_{\mu}=\mathcal{W}_{\tilde{\mathcal{H}}, a} \mathbb{M}_{\tilde{\mathcal{H}}^{-1 / 2}} \mathcal{W}_{\mathcal{H}_{0}, a}^{-1}$, where $\mathbb{M}_{\tilde{\mathcal{H}}^{-1 / 2}}: L^{2}\left(\mathcal{H}_{0}, a\right) \rightarrow L^{2}(\tilde{\mathcal{H}}, a)$ is the multiplication operator by $\tilde{\mathcal{H}}^{-1 / 2}$, that is, $\mathbb{M}_{\tilde{\mathcal{H}}^{-1 / 2}}: X \mapsto \tilde{\mathcal{H}}^{-1 / 2} X$. Since $\mathbb{M}_{\tilde{\mathcal{H}}^{-1 / 2}}$ is unitary, the operator $V_{\mu}$ is unitary as 
well. It is also clear that $V_{\mu} \mathrm{PW}_{r}=\mathcal{B}(\tilde{\mathcal{H}}, r)$ for every $r \in[0, a]$. Using Lemma 3.3. we see that $\mathcal{B}(\tilde{\mathcal{H}}, r)=\left(\mathrm{PW}_{r}, \mu\right)$, as required.

$(b) \Rightarrow(a)$. We will show that the function $\xi=\xi_{\mathcal{H}}$ is absolutely continuous. Let $\chi_{r}$ be the indicator function of an interval $[0, r]$. For every $r \in[0, a]$ consider the functions $X_{\xi(r)}=\chi_{\xi(r)}\left(\begin{array}{l}1 \\ 0\end{array}\right), Y_{\xi(r)}=\chi_{\xi(r)}\left(\begin{array}{c}0 \\ 1\end{array}\right)$ in $L^{2}(\mathcal{H}, \xi(r))$. A straightforward modification of Lemma 3.3 gives $\mathcal{B}(\mathcal{H}, \xi(r))=\left(\mathrm{PW}_{r}, \mu\right)$ for all $r \in[0, a]$. Put

$$
X_{r}^{0}=\mathcal{W}_{\mathcal{H}_{0}, a}^{-1} V_{\mu}^{-1} \mathcal{W}_{\mathcal{H}, a} X_{\xi(r)}, \quad Y_{r}^{0}=\mathcal{W}_{\mathcal{H}_{0}, a}^{-1} V_{\mu}^{-1} \mathcal{W}_{\mathcal{H}, a} Y_{\xi(r)} .
$$

Since $V_{\mu}$ is isometric and $V_{\mu} \mathrm{PW}_{r}=\left(\mathrm{PW}_{r}, \mu\right)$, we have $\mathcal{P}_{\mu, r} V_{\mu}=V_{\mu} \mathcal{P}_{r}$, where $\mathcal{P}_{r}$, $\mathcal{P}_{\mu, r}$ are the orthogonal projections on $\mathrm{PW}_{a},\left(\mathrm{PW}_{a}, \mu\right)$, with ranges $\mathrm{PW}_{r},\left(\mathrm{PW}_{r}, \mu\right)$, respectively. It follows that $X_{r}^{0}=\chi_{r} X_{a}^{0}$ and $Y_{r}^{0}=\chi_{r} Y_{a}^{0}$. Using the fact that the operators $\mathcal{W}_{\mathcal{H}_{0}, a}, \mathcal{W}_{\mathcal{H}, a}$ are unitary, we obtain

$$
\begin{aligned}
\int_{0}^{\xi(r)} \operatorname{trace} \mathcal{H}(t) d t & =\int_{0}^{\xi(r)}\left(\left\langle\mathcal{H}(t)\left(\begin{array}{c}
1 \\
0
\end{array}\right),\left(\begin{array}{l}
1 \\
0
\end{array}\right)\right\rangle+\left\langle\mathcal{H}(t)\left(\begin{array}{c}
0 \\
1
\end{array}\right),\left(\begin{array}{l}
0 \\
1
\end{array}\right)\right\rangle\right) d t \\
& =\left\|X_{\xi(r)}\right\|_{L^{2}(\mathcal{H}, \ell)}^{2}+\left\|Y_{\xi(r)}\right\|_{L^{2}(\mathcal{H}, \ell)}^{2} \\
& =\left\|X_{r}^{0}\right\|_{L^{2}\left(\mathcal{H}_{0}, a\right)}^{2}+\left\|Y_{r}^{0}\right\|_{L^{2}\left(\mathcal{H}_{0}, a\right)} \\
& =\left\|\chi_{r} X_{a}^{0}\right\|_{L^{2}\left(\mathcal{H}_{0}, a\right)}^{2}+\left\|\chi_{r} Y_{a}^{0}\right\|_{L^{2}\left(\mathcal{H}_{0}, a\right)}^{2} \\
& =\int_{0}^{r}\left(\left\|X_{a}^{0}(t)\right\|_{\mathbb{C}^{2}}^{2}+\left\|Y_{a}^{0}(t)\right\|_{\mathbb{C}^{2}}^{2}\right) d t
\end{aligned}
$$

The above equalities hold for all $r \in[0, a]$. Let us define the function $\kappa$ on $[0, \ell]$ by

$$
\kappa(s)=\int_{0}^{s} \operatorname{trace} \mathcal{H}(t) d t, \quad s \in[0, \ell] .
$$

Then $\kappa$ is an absolutely continuous function with positive derivative almost everywhere on $[0, \ell]$, hence the inverse mapping $\kappa^{-1}$ is also absolutely continuous and has positive derivative. On the other hand, formula (31) shows that $\kappa(\xi)$ is an absolutely continuous function. It follows that the superposition $\xi=\kappa^{-1}(\kappa(\xi))$ is absolutely continuous and hence $\operatorname{det} \mathcal{H}>0$ almost everywhere on $[0, \ell]$.

$(b) \Rightarrow(c)$. Since $\mu$ satisfies (1), the identical embedding $j: \mathrm{PW}_{a} \rightarrow\left(\mathrm{PW}_{a}, \mu\right)$ is a bounded and invertible operator. Define $A=V_{\mu}^{-1} j$. Then for all $f, g$ in $\mathrm{PW}_{a}$ we have

$$
\left(A^{*} A f, g\right)=\left(V_{\mu}^{-1} j f, V_{\mu}^{-1} j g\right)_{L^{2}(\mathbb{R})}=(j f, j g)_{L^{2}(\mu)}=\int_{\mathbb{R}} f \bar{g} d \mu=\left(T_{\mu, a} f, g\right),
$$

by the unitarity of the operator $V_{\mu}$. It follows that $T_{\mu, a}=A^{*} A$. By construction, the operator $A$ is invertible. We also have $A \mathrm{PW}_{r}=\mathrm{PW}_{r}$ for all $r \in[0, a]$, hence $A$ is upper-triangular.

$(c) \Rightarrow(b)$. Assume that $T_{\mu, a}$ admits a left triangular factorization $T_{\mu, a}=A^{*} A$. Define the operator $V_{\mu}: \mathrm{PW}_{a} \rightarrow\left(\mathrm{PW}_{\mu}, a\right)$ by $V_{\mu}=j A^{-1}$, where $j$ is the embedding from $\mathrm{PW}_{a}$ to $\left(\mathrm{PW}_{a}, \mu\right)$. Then $V_{\mu} \mathrm{PW}_{r}=\left(\mathrm{PW}_{r}, \mu\right)$ for every $r \in[0, a]$ and

$$
\begin{aligned}
\left(V_{\mu} f, V_{\mu} g\right)_{L^{2}(\mu)} & =\left(\left(A^{-1}\right)^{*} j^{*} j A^{-1} f, g\right)_{L^{2}(\mathbb{R})} \\
& =\left(\left(A^{*}\right)^{-1} T_{\mu, a} A^{-1} f, g\right)_{L^{2}(\mathbb{R})}=(f, g)_{L^{2}(\mathbb{R})},
\end{aligned}
$$

where we used the identity $T_{\mu, a}=j^{*} j$, see (32). Since $A$ and $j$ are invertible, $V_{\mu}$ is a unitary operator. 


\section{REFERENCES}

[1] Anton Baranov, Roman Bessonov, and Vladimir Kapustin. Symbols of truncated Toeplitz operators. J. Funct. Anal., 261(12):3437-3456, 2011.

[2] R. V. Bessonov and R. V. Romanov. An inverse problem for weighted Paley-Wiener spaces. preprint arXiv:1509.08117, 2015.

[3] Louis de Branges. Some Hilbert spaces of entire functions. ii. Trans. Amer. Math. Soc., 99:118-152, 1961.

[4] Louis de Branges. Hilbert spaces of entire functions. Prentice-Hall, Inc., Englewood Cliffs, N.J., 1968.

[5] Sergey A. Denisov. Continuous analogs of polynomials orthogonal on the unit circle and Kreĭn systems. IMRS Int. Math. Res. Surv., pages Art. ID 54517, 148, 2006.

[6] I. M. Gelfand and B. M. Levitan. On the determination of a differential equation from its spectral function. American Mathematical Society, 1955.

[7] I. C. Gohberg and M. G. Krein. Theory and Applications of Volterra Operators in Hilbert Space, volume 24 of Translations of Mathematical Monographs. American Mathematical Society, 1970.

[8] I. S. Kac and M. G. Krein. On the spectral functions of the string. Amer. Math. Soc. Transl, 103(2):19-102, 1974.

[9] M. G. Krein. On a fundamental approximation problem in the theory of extrapolation and filtration of stationary random processes. Dokl. Acad. Nauk SSSR, 94:13-16, 1954.

[10] M. G. Krein. Continuous analogues of propositions on polynomials orthogonal on the unit circle. Dokl. Akad. Nauk SSSR (N.S.), 105:637-640, 1955.

[11] David R. Larson. Nest algebras and similarity transformations. Annals of Mathematics, 121(2):409-427, 1985.

[12] Vladimir Aleksandrovich Marchenko. Sturm-Liouville operators and applications, volume 373. American Mathematical Soc., 2011.

[13] I. P. Natanson. Theory of functions of a real variable, volume 1. Ungar, New York, 1964. Translated by L. F. Boron, with the editorial collaboration of and with annotations by E. Hewitt.

[14] Nikolai K. Nikolski. Operators, functions, and systems: an easy reading. Vol. 1, volume 92 of Mathematical Surveys and Monographs. American Mathematical Society, Providence, RI, 2002. Hardy, Hankel, and Toeplitz, Translated from the French by Andreas Hartmann.

[15] Roman Romanov. Canonical systems and de Branges spaces. preprint arXiv:1408.6022, 2014.

[16] Lev A. Sakhnovich. On triangular factorization of positive operators. In Recent Advances in Matrix and Operator Theory, pages 289-308. Springer, 2007.

[17] Lev A. Sakhnovich. Effective construction of a class of positive operators in Hilbert space, which do not admit triangular factorization. In Levy Processes, Integral Equations, Statistical Physics: Connections and Interactions, pages 85-99. Springer, 2012.

[18] Donald Sarason. Algebraic properties of truncated Toeplitz operators. Oper. Matrices, 1(4):491-526, 2007.

[19] V. I. Vasyunin. The sharp constant in the reverse Hölder inequality for the Muckenhoupt weights. Algebra i Analiz, 15(1):73-117, 2003.

[20] Henrik Winkler. Operator Theory, chapter Two-Dimensional Hamiltonian Systems, pages 1-22. Springer Basel, Basel, 2014.

St.Petersburg State University (7/9, Universitetskaya nab., St.Petersburg, 199034 Russia) and St.Petersburg Department of Steklov Mathematical Institute of Russian Academy of Science (27, Fontanka, St.Petersburg, 191023 Russia)

E-mail address: bessonov@pdmi.ras.ru 